\title{
Control of a Jugular Bulb Bleeding in Endoscopic Ear Surgery: a Case Report
}

\author{
Yousef Aljathlany ${ }^{1}$ and Jihad Nassar ${ }^{2 *}$ \\ ${ }^{1}$ Senior Resident, Department of Otolaryngology Head and Neck Surgery, King Abdulaziz University Hospital, Kingdom of Saudi Arabia \\ ${ }^{2}$ Consultant, Department of Surgery, Division of ENT, King Abdulaziz Medical City, National Guard Health Affairs, Kingdom of Saudi Arabia
}

Submission: August 20, 2017; Published: August 28, 2017

*Corresponding author: Jihad Nassar, Department of Surgery-Division of ENT, King Abdulaziz Medical City - Ministry of National Guard - Health affairs, P.O.Box 22490; Mail box: 1446, Riyadh 11426, Saudi Arabia, Tel: +966559319977; Email: jihadnassar@me.com

\begin{abstract}
Endoscopic ear surgery (EES) is gaining more popularity and applications since late 90 's. Little was published about the complications and their endoscopic management including major intra-operative bleeding. We are reporting a case of management of bleeding from a high jugular bulb during an endoscopic Transcanal tympanoplasty without the need to a microscopic conversion. To the best of our knowledge; this is the first case of a major intra-operative bleeding in middle ear surgery that was controlled solely with the endoscope.

Keywords: Endoscopic ear surgery; Jugular bulb; Bleeding; Tympanoplasty

Abbreviations: CT: Computed Tomography; EES: Endoscopic Ear Surgery; JB: Jugular Bulb
\end{abstract}

\section{Introduction}

The concept of endoscopy in middle ear surgery started at 1967 by Mer et al. [1] for examining the middle ear. In the late $90 \mathrm{~s}$, the use of endoscope was shifted from a diagnostic tool or simple assisting tool for the microscope to be the sole instrument in middle ear surgery when Tarabichi published 2 case series in 1997 and 1999 performing fully endoscopic ear surgeries [2,3]. Since then, Endoscopic Ear Surgery (EES) has gained increasing popularity throughout the world and several studies came out to support it. However, most of these studies were describing the techniques, the indications and comparison to the conventional microscopic surgery, with a minority addressing the complications and its endoscopic management [4]. One of the poorly described complications in EES is the major intraoperative bleeding where few cases have been reported [4]. Dehiscent high jugular bulb (HJP) is a source of major bleeding in middle ear surgery in spite of its relative rarity. We are reporting a case of a major bleeding from jugular bulb encountered during a tympanoplasty case that was managed endoscopically without the need to shift to microscopic surgery. To the best of our knowledge, this is the first reported case of jugular bulb bleeding during endoscopic tympanoplasty, solely managed endoscopically.

\section{Case Report}

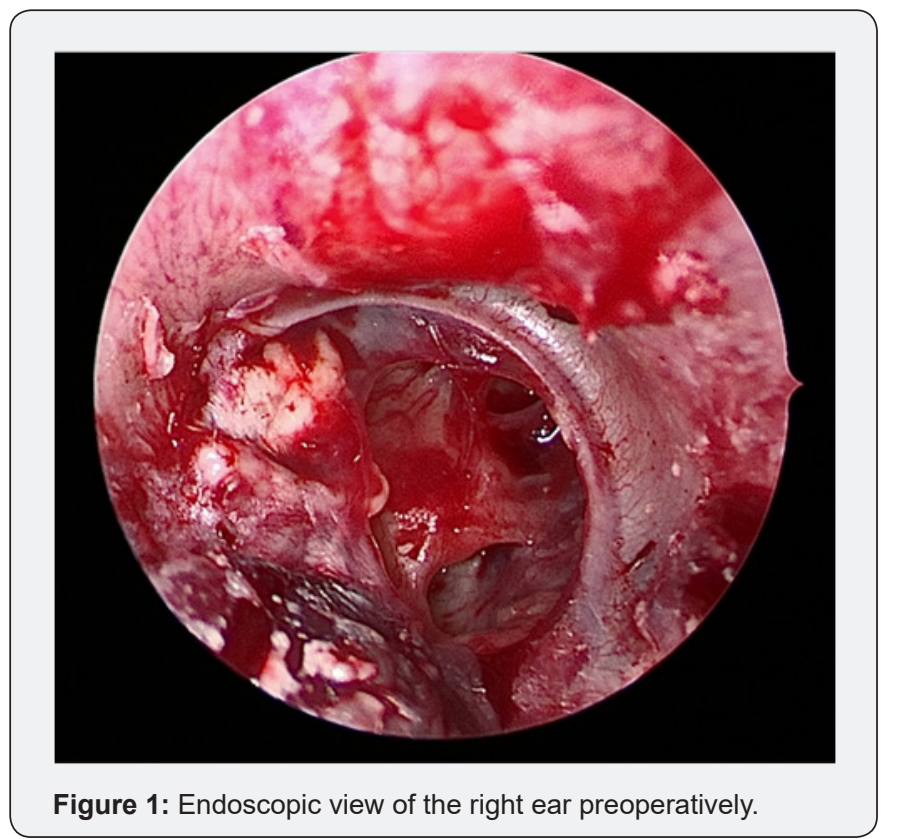

A 24-year-old gentleman referred to our clinic from the primary health care with a history of bilateral decrease hearing since childhood, associated with recurrent bilateral ear discharge up to 3 years prior to his presentation. He has occasional non- 


\section{Global Journal of Otolaryngology}

pulsatile tinnitus bilaterally, and denies any history of vertigo, aural fullness or facial weakness. He was healthy otherwise and had no previous ear surgery. On examination, he had bilateral dry subtotal perforations with inferior myringosclerotic patch in the right ear (Figure 1). Weber test lateralized to the right side and Rinne test was negative bilaterally. Pure tone audiometry showed bilateral mild to moderate conductive hearing loss with slightly worse thresholds on the right side. The decision was made to operate on the right ear first and the patient gave consent for an endoscopic transcanal tympanoplasty (Video).

The senior author was the primary surgeon. Intraoperatively, while elevating of the tympano-meatal flap and entering the middle ear, a gush of blood was encountered at the level of the bony annulus inferiorly at around 8 0'clock position. The bleeding was profuse indicating a high jugular bulb bleeding (Figure 2). The bleeding was first controlled by applying pressure over its source at bony annulus using the suction (Figure 3). While keeping pressure over the bleeding point, the assistant surgeon introduced pieces of oxidized cellulose Surgicel $^{\mathrm{TM}}$ (Ethicon, Inc., a Johnson \& Johnson company; Somerville, NJ) (Figure 4). The bleeding stopped after few small pieces of surgicel and pressure-suction over a neuropatty. The tymapnoplasty was aborted and the external auditory canal was packed with gel foam (Figure 5). The amount of bleeding was estimated around $50 \mathrm{ml}$.

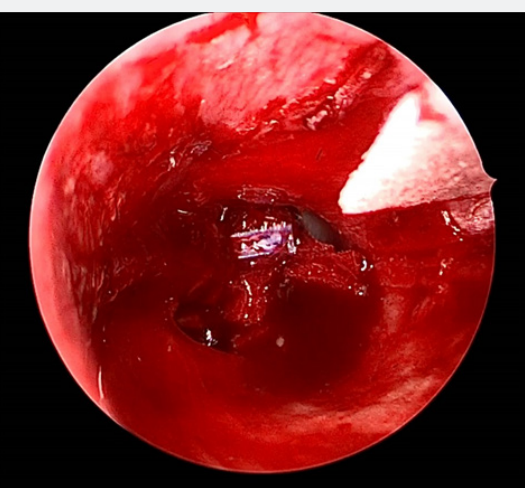

Figure 2: A gush of bleeding at the inferior bony sulcus can be observed.

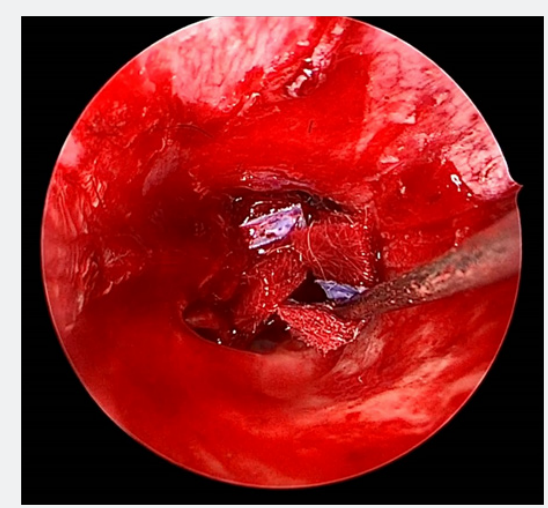

Figure 3: Control of bleeding initially by applying pressure with the suction.
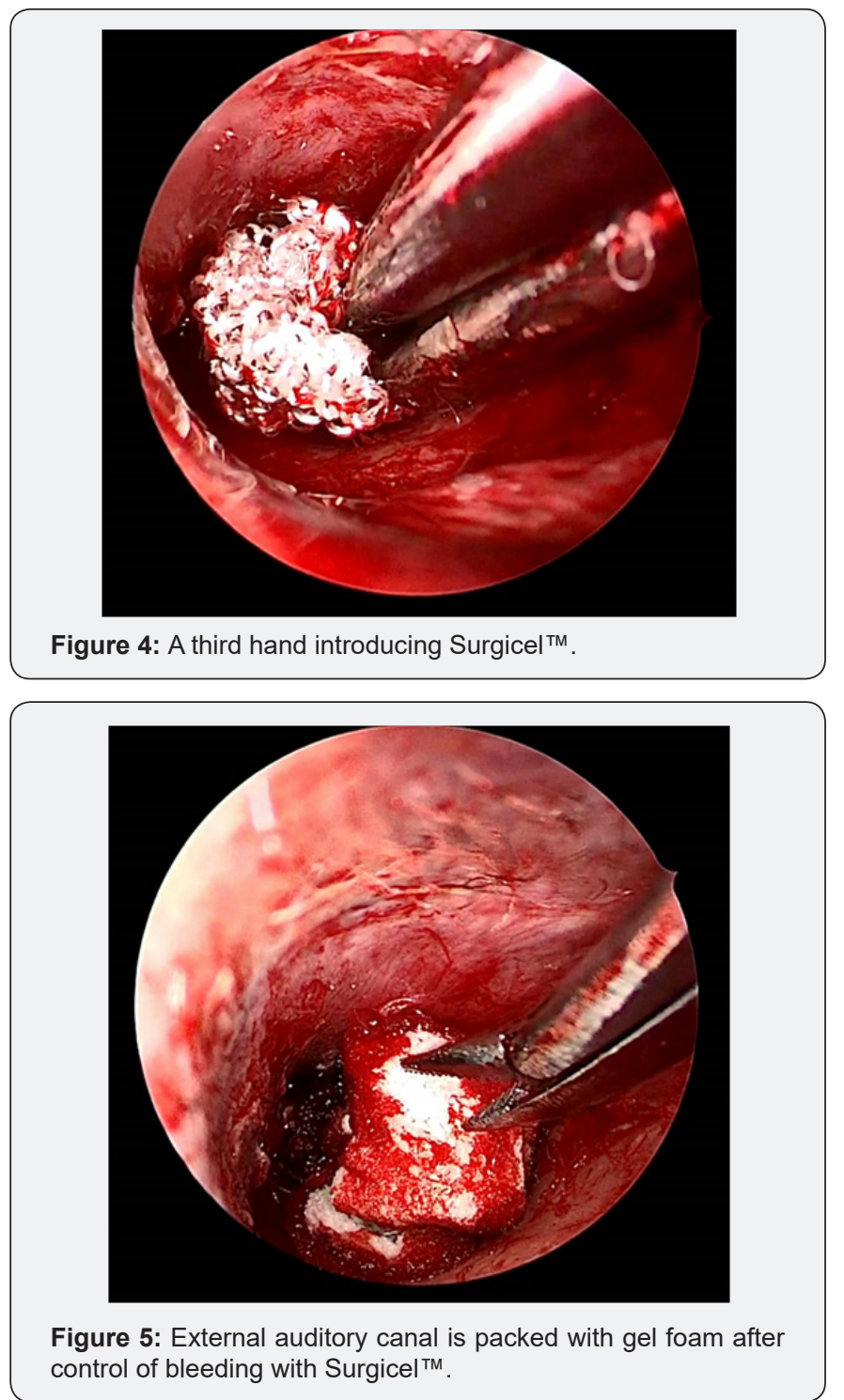

The patient was successfully awakened from the anesthesia, extubated, and shifted to the recovery room in a stable condition. Post-operative weber was lateralizing to the operated ear. He was discharged home within 24 hours and instructed to use antibiotic ear drops and to avoid any aggressive activity. He had no post-operative dizziness. The patient was then seen 2 weeks later in the clinic, reported no bleeding episodes or dizziness. 
Examination showed a dry ear with some remnants of gel foam and his weber test was still lateralizing to the operated ear.

\section{Discussion}

Since the beginning of endoscopic ear surgery, multiple studies have been published comparing the EES to the microscopic ear surgery but minority of them were addressing the complications and their management endoscopically [4]. Major intra-operative bleeding was seldom described in EES. One case of intra-operative bleeding was reported by Hunter et al when they encountered excessive bleeding during an endoscopic stapes surgery and they had to convert to the microscopicassisted surgery to control the bleeding [5]. However, in our case we were able to manage a profuse bleeding endoscopically without microscopic conversion. Major vascular bleeding in chronic ear surgery is mostly due to sigmoid sinus, jugular bulb or internal carotid artery (ICA) injury. Internal carotid artery dehiscence can be seen in $1 \%$ of the bony plate in the middle ear and it is exceedingly rare to be prone for injury and bleeding $[6,7]$.

Sigmoid sinus bleeding is mostly encountered in mastoid surgery and rarely during tympanoplasty or stapes surgery [8]. Jugular bulb bleeding in middle ear surgery (excluding mastoidectomy) is relatively higher compared to the one resulting from the aberrant carotid artery or sigmoid sinus injury. The first reported case of jugular bulb bleeding was by Page in 1914 on a 10 months old girl during myringotomy procedure [9]. Radiologically, a study looking into 2020 temporal bone computed tomographies (CT) showed a high jugular bulb in $15 \%$ and dehiscence in $7.5 \%$ [10]. The high risk of bleeding was defined by Atmaca et al to be a high and dehiscent jugular bulb which was observed in only $2 \%$ of the temporal bone CT's [10].

Clinically, Huang et al retrospectively reviewed 1657 patients of middle ear cleft diseases who underwent surgery over a 20 years period, and found only 10 ears $(0.5 \%)$ in 9 patients with dehiscent high jugular bulb. Four out of the nine patients had tinnitus and only 2 of them had vertigo. In all cases the dehiscent high jugular bulb was discovered intra-operatively. Only two cases encountered bleeding (around $30 \mathrm{ml}$ each) that were controlled using gel foam [11]. Our patient had occasional tinnitus bilaterally that was non-pulsatile, and presented with a dry tympanic membrane perforation for more than 3 years, hence a preoperative imaging was not considered. In our practice, we only consider preoperative imaging in continuously draining ears and suspected cholesteatoma or tumors. No alarming signs or symptoms of vascular anomalies or a dehiscent high jugular bulb were suspected.

Intraoperatively, a high jugular bulb was identified as minor with no dehiscence. The bleeding occurred while elevating the annulus postero-inferiorly using a needle pick. The gush of bleeding was suspicious as originating from the jugular bulb. Pressure with the tip of suction confirmed its source at the inferior bony annulus apparently abutting a dehiscent high jugular bulb at this site. The bleeding was controlled endoscopically with the help of an assistant surgeon (a senior resident) providing a third hand. EES has been criticized being a one-handed technique. It has also been suggested in the literature that in the event of excessive bleeding, one should consider conversion to microscopic surgery [12].

We argue that with the help of an assistant surgeon, one can achieve the bleeding control endoscopically and overcome the need for microscopic conversion. The senior author often considers a third hand to help out the residents with suction for training purposes. Although a scope holder has been recently developed, we still prefer to hold the scope because of the frequent need to change its position, clean the lens and prevent overheating. We believe that with slight modifications of the instruments, proper training of residents and increasing familiarity of nurses with the EES approach, as well as adopting some tips in minimizing bleeding would overcome the shortcomings of endoscopic ear surgery being a one-handed technique.

\section{Conclusion}

Bleeding from a high jugular bulb is relatively rare but serious complication, it may occur in what seems to be a straightforward middle ear case. If encountered during endoscopic ear surgery it can be managed without microscopic conversion particularly with the presence of an assistant surgeon who can offer a third hand in similar incidents. With proper training and experience, surgeons are becoming more confident and less likely to convert to microscopic assistance in challenging events in EES.

\section{References}

1. Mer SB, Derbyshire AJ, Brushenko A, Pontarelli DA (1967) Fiberopticendotoscopes for examining the middle ear. Archives of Otolaryngology 85(4): 387-393.

2. Tarabichi M (1997) Endoscopic management of acquired cholesteatoma. Am J Otol 18: 544-549.

3. Tarabichi M (1999) Endoscopic middle ear surgery. Annals of Otology, Rhinology \& Laryngology 108(1): 39-46.

4. Kozin ED, Gulati S, Kaplan AB, Lehmann AE, Remenschneider, et al. (2015) Systematic review of outcomes following observational and operative endoscopic middle ear surgery. The Laryngoscope 125(5): 1205-1214.

5. Hunter JB, Zuniga MG, Leite J, Killeen D, Wick C, et al. (2016) Surgical and audiologic outcomes in endoscopic stapes surgery across 4 institutions. Otolaryngology-Head and Neck Surgery 154(6): 10931098.

6. Myerson MC, Rubin H, GilbertJG (1934) Anatomic studies of the petrous portion of the temporal bone. Archives of Otolaryngology 20(2): 195210 .

7. Goldman NC, Singleton GT, Holly EH (1971) Aberrant internal carotid artery: presenting as a mass in the middle ear. Archives of Otolaryngology 94(3): 269-273.

8. Ulug T, Basaran B, Minareci O, Aydin K (2004) An unusual complication 
of stapes surgery: profuse bleeding from the anteriorly located sigmoid sinus. European Archives of Oto-Rhino-Laryngology and Head \& Neck 261(7): 397-399.

9. Page JR (1914) XIX. A Case of Probable Injury to the Jugular Bulb following Myringotomy in an Infant Ten Months Old. Annals of Otology, Rhinology \& Laryngology 23(1): 161-163.

10. Atmaca S, Elmali M, Kucuk H (2014) High and dehiscent jugular bulb: clear and present danger during middle ear surgery. Surgical and Radiologic Anatomy 36(4): 369-374.

11. Huang BR, Wang CH, Young YH (2006) Dehiscent high jugular bulb: a pitfall in middle ear surgery. Otology \&Neurotology 27(7): 923-927.

12. Lakpathi G, Reddy LS (2016) Comparative Study of Endoscope Assisted Myringoplasty and Microscopic Myringoplasty. Indian Journal of Otolaryngology and Head \& Neck Surgery 68(2): 185-190.

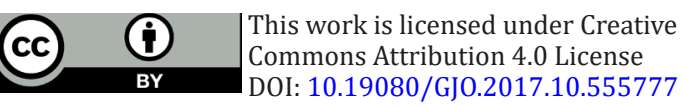

